\title{
Impact of the SARS-CoV2 pandemic dissemination on the management of neuroendocrine neoplasia in Italy: a report from the Italian Association for Neuroendocrine Tumors (Itanet)
}

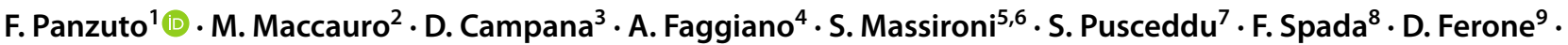 \\ R. Modica ${ }^{10} \cdot$ C. M. Grana ${ }^{11} \cdot$ P. Ferolla ${ }^{12} \cdot$ M. Rinzivillo ${ }^{1} \cdot$ G. Badalamenti ${ }^{13} \cdot$ M. C. Zatelli ${ }^{14} \cdot$ F. Gelsomino $^{15}$. \\ E. De Carlo ${ }^{16} \cdot$ M. Bartolomei ${ }^{17} \cdot$ M. P. Brizzi ${ }^{18} \cdot$ S. Cingarlini ${ }^{19} \cdot$ A. Versari ${ }^{20} \cdot$ G. Fanciulli ${ }^{21}$ - E. Arvat ${ }^{22} \cdot$ E. Merola $^{23}$. \\ M. Cives $^{24} \cdot$ S. Tafuto $^{25} \cdot$ S. Baldari ${ }^{26} \cdot$ M. Falconi ${ }^{27}$
}

Received: 17 June 2020 / Accepted: 9 August 2020 / Published online: 16 August 2020

(c) Italian Society of Endocrinology (SIE) 2020

\begin{abstract}
Introduction The organization of the healthcare system has significantly changed after the recent COVID-19 outbreak, with a negative impact on the management of oncological patients. The present survey reports data collected by the Italian Association for Neuroendocrine Tumors on the management of patients with neuroendocrine neoplasia (NEN) during the pandemic dissemination.

Methods A survey with 57 questions was sent to NEN-dedicated Italian centers regarding the management of patients in the period March 9, 2020, to May 9, 2020

Results The main modification in the centers' activity consisted of decreases in newly diagnosed NEN patients $(-76.8 \%)$, decreases in performed surgical procedures $(-58 \%)$, delays to starting peptide receptor radionuclide therapy $(45.5 \%)$, postponed/canceled follow-up examinations (26\%), and canceled multidisciplinary teams' activity (20.8\%). A low proportion of centers $(<10 \%)$ reported having to withdraw systemic anti-tumor medical treatment due to concerns about the pandemic situation, whereas PRRT was withdrawn from no patients.

Conclusion Although the COVID-19 outbreak induced the centers to reduce some important activities in the management of NEN patients, the Italian network was able to provide continuity in care without withdrawing anti-tumor treatment for the majority of patients.
\end{abstract}

Keywords COVID-19 $\cdot$ SARS-CoV2 $\cdot$ Pandemic $\cdot$ Neuroendocrine tumors $\cdot$ Multidisciplinary team $\cdot$ Management $\cdot$ Peptide receptors radionuclide therapy

\section{Introduction}

Following the spread of the COVID-19 outbreak in Italy in February 2020 induced by the novel SARS-CoV2 virus, strict measures have been implemented by the national government, including restricting movement and recommendations to go to hospitals only in cases of absolute necessity.

Electronic supplementary material The online version of this article (https://doi.org/10.1007/s40618-020-01393-4) contains supplementary material, which is available to authorized users.

F. Panzuto

fpanzuto@ospedalesantandrea.it

Extended author information available on the last page of the article
The healthcare system has suffered from the increasing numbers of infected persons requiring intensive care, and hospitals' activities have been modified to satisfy the novel emerging need [1-3]. Oncological patients have been reported to be at higher risk of being affected by COVID-19, with an increased risk of severe illness and mortality $[4,5]$. Several reports have been produced evaluating the potential impact of the SARS-CoV2 pandemic dissemination on the management of patients with different kinds of tumors, including colon, breast, and lung cancers [6-8]. However, no data are available for neuroendocrine neoplasia (NENs). Given the rarity of these diseases and the need for dedicated specialized centers to care for NEN patients, collecting data in this specific patient population may be challenging. 
The aim of this study was to understand how the pandemic dissemination of SARS-CoV2 infection has modified the clinical management of NEN patients in Italy.

\section{Methods}

An invitation to participate in an online survey (COVINET survey) by the SurveyMonkey ${ }^{\circledR}$ platform (https://it.surve ymonkey.com/) was sent to the members of the Italian Association for Neuroendocrine Tumours (Itanet) (www.ita-netorg) using email distribution. Itanet is a national multidisciplinary NEN-dedicated scientific association comprised of approximately 200 physicians, including virtually all of the Italian specialists involved in the management of NEN patients. The questionnaire for the online survey had been prepared by a team including oncologists (SP, FS), gastroenterologists (FP, SM), an endocrinologist (AF), an internal medicine physician (DC), a surgeon (MF) and a nuclear medicine (MM) physician on behalf of Itanet, and was sent to the Society's members with specific instructions on how to answer.

A single member of each receiving center was identified as the referral survey sender to avoid the risk of potential duplicates. Specifically, each sender was required to collect data from the center after involving the components of the NEN dedicated team. The median number of physicians working in each NEN dedicated multidisciplinary teams was 6 , thus resulting in an overall amount of physicians involved in the survey of approximatively 140 specialists. The final analysis of collected data was performed by the study coordinator (FP), and shared with the member of the Itanet board (MF, SP, FS, SM, AF, DC) who reviewed and approved the results. The survey consisted of 57 questions related to the clinical management of NEN patients during the 2 months from March 9, 2020 (the beginning of the "lock-down" period in Italy in accordance with the national legislation), to May 9, 2020 (defined as the observation period) (supplementary file 1). It included three main sections: (i) features and standard activity of the center before the SARS-CoV2 dissemination; (ii) modality used by the center to follow NEN patients during the reference period; (iii) estimation of the modification/delay/reduction of the planned clinical activity, with particular effects on patients' treatment. Data were collected by the SurveyMonkey ${ }^{\circledR}$ platform. This study was notified to the Ethics Committee of the coordinating center (Sant'Andrea University Hospital, ref. CE 7023_2020) and was carried out according to the Declaration of Helsinki.

\section{Results}

\section{Centers features and standard activity}

A total of 24 centers participated in the COVINET survey, including 6 Centers of Excellence for NEN management certified by the European Neuroendocrine Tumors Society (ENETS). Of these, 15 (62.5\%) were located in northern Italy, which was the area with a higher SARS-CoV2 incidence. The majority of the participating centers $(n=20,83.3 \%)$ were in hospitals that were included in the national network for COVID-19 management. As far as the volume of activity is concerned, 5 centers $(20.8 \%)$ were considered "low-activity centers" with $<100$ NEN patients in follow-up, 10 centers (41.7\%) had "mid-activity" with 100-300 NEN patients in active follow-up and the remaining 9 centers $(37.5 \%)$ were "high-activity centers" with $>300$ NEN patients followed. The mean reported number of planned visits to be performed for NEN outpatients during the observation period was 84 . All centers had a multidisciplinary team dedicated to the management of the NEN patients.

\section{Follow-up of NEN patients during the SARS-CoV2 pandemic dissemination}

Most centers ( $n=17,70.8 \%)$ modified patients' follow-up modality due to the pandemic dissemination. Specifically, in one center, both outpatients' visits and hospitalizations were stopped, whereas in $16(66.7 \%)$, they were reduced and limited to urgent clinical conditions. No change in the standard activity was reported by the remaining 7 centers $(29.2 \%)$. Multidisciplinary teams' activity was maintained in 19 centers (79.2\%); however, in 12 centers (50\%) the modality of discussion was modified by avoiding physical meetings in favor of web-based virtual meetings. In 5 centers (20.8\%), MDT activity was suspended, and meetings were not held. A significant decrease in ambulatory activity was reported during the observation period, with the mean number of visits for NEN outpatients being 30 (vs 84 planned before the COVID-19 outbreak, $-64.3 \%)$. Most centers $(n=21,87.5 \%)$ used telemedicine to follow-up NEN patients during the observation period, either by phone, video calls, email or instant messaging.

A large proportion of centers $(n=22,91.7 \%)$ reported that planned imaging follow-up procedures were rescheduled, and in $59 \%$ of patients, they were performed outside the center, where the patients were usually followed.

\section{Modification of center activity during the SARS-CoV2 pandemic dissemination}

There was a dramatic reduction in the centers' clinical activity (Table 1). The number of surgical procedures for 
Table 1 Consequences of COVID-19 dissemination on NENs management reported by the centers

\begin{tabular}{ll}
\hline Activity & $\begin{array}{l}\text { Reported } \\
\text { change } \\
(\%)\end{array}$ \\
\hline Decrease in newly diagnosed NEN & -76.8 \\
Suspended MDT activity & 20.8 \\
Decrease in surgical procedures & -58 \\
Delay to initiate PRRT & 45.5 \\
Delayed/cancelled follow-up examinations & 26 \\
\hline
\end{tabular}

NEN patients fell from 69 planned operations to 29 performed procedures (-58\%). A total of 109 NEN patients were newly diagnosed in the 24 participating centers during the observation period, again confirming a decrease of $76.8 \%$ compared with standard activity, during which the usual overall reported number of newly diagnosed patients by the centers was $235 /$ month. Among the newly diagnosed patients, $85.1 \%$ had non-functioning tumors and $56.4 \%$ had advanced metastatic disease.

When asking the center to estimate what was the percentage of reduction in clinical activity for NEN patients, 15 centers $(62.5 \%)$ reported a $25-50 \%$ reduction, and 7 centers $(29.2 \%)$ a decrease $>75 \%$. Only 2 centers $(8.3 \%)$ reported no significant decrease in clinical activity for NEN patients.

As far as medical treatments were concerned, the management of patients varied depending on the specific therapy. Specifically, somatostatin analogs therapy was maintained unchanged for all patients. Everolimus, sunitinib and chemotherapy were continued for $90.9 \%, 95.2 \%$, and $90.9 \%$ of patients, respectively.

As far as peptide receptor radionuclide therapy (PRRT) was concerned, 10 out of 24 participating centers (41.7\%) were qualified to perform PRRT by the National Health System. Overall, $45.5 \%$ of centers reported a delay in the initiation of PRRT for new candidates for treatment due to the pandemic situation. In those patients who had already started PRRT before the SARS-CoV2 dissemination, the administration schedule was not modified in $85 \%$ of cases, whereas in the remaining $15 \%$ of patients, it was delayed. However, PRRT was withdrawn from no patients. A proportion of $23.8 \%$ of centers reported concerns expressed by patients receiving PRRT regarding the safety of the treatment; however, only one single center asserted that patients decided not to initiate PRRT due to safety reasons.

Almost $1 / 4$ of centers $(21.7 \%$ ) referred patients who were candidates to begin PRRT to other centers, due to the limitations/patients' concerns due to the pandemic dissemination. A total of $13 \%$ of centers reported having referred to other centers patients who had already started PRRT.
A total of 12 centers $(50 \%)$ reported having stopped patients' enrollment in clinical trials or having experienced delays in activating new planned clinical trials.

When asked what was the most frequent general consequence of the pandemic dissemination on the management of NEN patients, the first three answers reported by the centers were: delay of diagnosing new NEN patients (50\%), delay of beginning previously planned treatment (37.7\%), and delay of performing planned follow-up examinations (26\%).

\section{Discussion}

In recent months, the COVID-19 pandemic dissemination has had a significant impact on hospital activity and organization, with several departments involved in the management of COVID-19 patients, including those usually dedicated to NEN patients.

Recently, suggestions on how health care providers might make modifications to their care of NEN patients has been proposed by the North American Neuroendocrine Tumor Society [9]. Furthermore, several studies performed on different kinds of cancers have clearly shown that this unexpected condition has negatively modified the level of caring for oncological patients [6-8]. To the best of our knowledge, this is the first report from a multidisciplinary community of physicians dedicated to caring for NEN patients evaluating the impact of the COVID-19 pandemic dissemination on the management of these patients.

Given the rarity and the peculiar indolent behavior of the majority of NENs, specific challenging issues need to be addressed by physicians dealing with these diseases, including (i) to maintain active MDT, which is well known to be a key factor in the effective care of NEN patients [10, 11]; (ii) to avoid delays in initial NEN diagnosis, which is frequently reported in real-world practice even before the pandemic dissemination [12]; (iii) to evaluate the potential increased risk of severe illness in NEN patients with COVID-19 receiving targeted agents with immunomodulatory activity such as everolimus and sunitinib; (iv) to maintain the scheduled workflow for patients on treatment or who are candidates for PRRT, which requires specific logistic organization in terms of timing of hospitalization; and (v) to carry out planned surgical procedures, which may provide definitive disease cures in NEN patients with localized or locally advanced diseases, as well as in selected cases of metastatic patients [13].

The main figures reported by the present survey confirm that a significant proportion of Italian NEN-dedicated centers experienced difficulties when facing these issues. The number of new diagnoses decreased by $76.8 \%$, resulting in a significant number of patients with undiagnosed NEN who will receive a delayed diagnosis after the emergency status 
resolves. This delay will have a negative impact by reducing their probability of receiving curative surgery for early-stage disease and by delaying the initiation of an effective antitumor medical therapy in those patients with more advanced disease. The significant decrease in newly diagnosed NEN patients may be related to several factors: (i) Refusal or delay of patients to come to hospitals, which is reasonable given the usual clinically indolent course of most non-functioning NENs, particularly in the early stage disease (notably, 56.4\% of newly diagnosed patients were reported wo have advanced metastatic disease when referring to the center, and $85.1 \%$ were non-funcioning); (ii) Reduction of the overall numbers of radiological procedures performed during the observation period, which might explain the decrease in number of incidentally found NEN that represent a consistent proportion of newly diagnosed NEN in general [14]; (iii) A general decrease in all cancer-related patients encounters which has recently been observed as a result of pandemic in other oncological settings [15].

Approximatively $1 / 4$ of participating centers $(26 \%)$ reported the need to postpone or cancel planned follow-up examinations. This figure is quite expected, given the reasonable need to minimize patients' access to the hospitals, a suggestion proposed by the oncologist community as a part of the specific care of oncological patients during the COVID-19 pandemic [16].

As far as PRRT is concerned, it was observed that the modality and timing of the treatment was maintained by almost all centers (85\%) despite the COVID-19 pandemic, suggesting that centers with more experience and with a dedicated nuclear medicine department performing PRRT were able to promptly adapt to the new indications and restrictions imposed by the extraordinary situation. Conversely, almost half of centers $(45.5 \%)$ reported that PRRT initiation was postponed in candidate patients who had not yet received their first dose of lutetium-177-DOTA-octreotate. This apparent discrepancy may be explained by the need to conserve health system resources for those patients who had already started PRRT to ensure treatment completion by deciding to delay treatment initiation in some patients, a figure that has recently been reported for other oncological diseases by the nuclear medicine community [17]. However, PRRT was withdrawn from no patients.

A significant decrease in NEN dedicated surgical procedures was reported (-58\%) in the survey. Deferring surgery for oncological disease may be a difficult choice for physicians, given the potential risk of tumor progression. However, in the era of COVID-19 that we are experiencing, this risk needs to be balanced with the potential increased risk of infection and severe illness that may occur as a consequence of immunodepression induced by the cancer itself and by surgical procedures [5]. Since most NEN are slow-growing, it seems reasonable to defer major surgical procedures with the intent to offer patients effective and safe management, as proposed for other nonendocrine early-stage cancers [18]. This may explain the choice of the majority of centers to defer planned surgery in a significant proportion of NEN patients.

An additional finding reported by the present survey was the low proportion of patients receiving medical treatments for whom a schedule modification was proposed. Although this is highly expected for SSAs, which have a negligible impact on the immune system, it seems less obvious for everolimus, sunitinib, and chemotherapy, which were maintained in $90.9 \%, 95.2 \%$, and $90.9 \%$ of cases. Regarding everolimus and sunitinib, to date there is no available scientific data to compare this figure with other reports even outside the setting of NEN patients. As far as chemotherapy is concerned, other surveys performed of nonendocrine cancers have reported a low proportion of oncologists who changed treatment plans in their clinical practice $[19,20]$. This figure is in agreement with data reported by the present survey, confirming the ability of the NEN dedicated teams to continue to provide optimal care to their patients.

\section{Conclusion}

The spread of the COVID-19 pandemic had a significant impact on the management of NEN patients, reducing the number of newly diagnosed patients, deferring a significant proportion of surgical procedures, and delaying the beginning of PRRT in a consistent proportion of candidate patients.

However, the network of NEN dedicated Italian centers was able to provide continuity in care without withdrawing medical treatments or PRRT for the majority of patients. A better understanding of the real consequences of the COVID-19 pandemic on the care of NEN patients will be feasible once the emergency resolves.

Funding This research did not receive any specific grant from funding agencies in the public, commercial, or not-for-profit sectors.

\section{Compliance with ethical standards}

Conflict of interest Authors have no conflict of interest to declare.

Ethical approval All procedures were approved by the Coordinating Center (Sant'Andrea University Hospital of Rome).

Informed consent All participant members provided consent prior to their participation. 


\section{References}

1. Isidori AM, Jannini EA, Lenzi A, Ghigo E (2020) Board of Full Professors in Endocrinology, Directors of the Endocrinology, Metabolic Disorders Residency Program. The Italian EndoCOVID-19. Our response to the emergency. J Endocrinol Invest. https://doi.org/10.1007/s40618-020-01279-5 (published online ahead of print, 2020 May 8 )

2. Mistretta FA, Luzzago S, Molendini LO et al (2020) A guide for oncologic patient management during Covid-19 pandemic: the initial experience of an Italian oncologic hub with exemplificative focus on uro-oncologic patients. Cancers (Basel) 12(6):E1513. https://doi.org/10.3390/cancers 12061513 (Published 2020 Jun 10)

3. Maida M, Sferrazza S, Savarino E et al (2020) Impact of the COVID-19 pandemic on gastroenterology divisions in Italy: a national survey. Dig Liver Dis. https://doi.org/10.1016/j. dld.2020.05.017 (published online ahead of print, 2020 May 16)

4. Liang W, Guan W, Chen R et al (2020) Cancer patients in SARSCoV-2 infection: a nationwide analysis in China. Lancet Oncol 21(3):335-337

5. Wang H, Zhang L (2020) Risk of COVID-19 for patients with cancer. Lancet Oncol 21(4):e181

6. Pellino G, Spinelli A (2020) How COVID-19 outbreak is impacting colorectal cancer patients in Italy: a long shadow beyond infection. Dis Colon Rectum. https://doi.org/10.1097/DCR.00000 00000001685 (published online ahead of print, 2020 Mar 17)

7. Corsi F, Caruso A, Albasini S et al (2020) Management of breast cancer in an EUSOMA-accredited breast unit in Lombardy, Italy, during the COVID-19 pandemic. Breast J. https://doi.org/10.1111/ tbj.13926.10.1111/tbj.13926 (published online ahead of print, 2020 May 30)

8. Xu Y, Liu H, Hu K, Wang M (2020) Clinical recommendations on lung cancer management during the COVID-19 pandemic. Thorac Cancer. https://doi.org/10.1111/1759-7714.13498 (published online ahead of print, 2020 May 29)

9. Bergsland EK, Halperin DM, Dillon JS et al (2020) North American neuroendocrine tumor society guide for neuroendocrine tumor patient health care providers during COVID-19. Pancreas. https ://doi.org/10.1097/MPA.0000000000001561 (published online ahead of print, 2020 May 19)

10. Magi L, Mazzuca F, Rinzivillo M et al (2019) Multidisciplinary management of neuroendocrine neoplasia: a real-world experience from a referral center. J Clin Med 8(6):910. https://doi. org/10.3390/jcm8060910 (Published 2019 Jun 25)

11. Grimaldi F, Fazio N, Attanasio R et al (2014) Italian Association of Clinical Endocrinologists (AME) position statement: a stepwise clinical approach to the diagnosis of gastroenteropancreatic neuroendocrine neoplasms. J Endocrinol Invest 37:875-909

12. Basuroy R, Bouvier C, Ramage JK, Sissons M, Kent A, Srirajaskanthan R (2018) Presenting symptoms and delay in diagnosis of gastrointestinal and pancreatic neuroendocrine tumours. Neuroendocrinology 107(1):42-49. https://doi.org/10.1159/000488510

13. Cives M, Strosberg JR (2018) Gastroenteropancreatic neuroendocrine tumors. CA Cancer J Clin 68(6):471-487. https://doi. org/10.3322/caac.21493

14. Partelli S, Giannone F, Schiavo Lena M et al (2019) Is the real prevalence of pancreatic neuroendocrine tumors underestimated? a retrospective study on a large series of pancreatic specimens. Neuroendocrinology 109(2):165-170. https://doi. org/10.1159/000499606

15. London JW, Fazio-Eynullayeva E, Palchuk MB, Sankey P, McNair C (2020) Effects of the COVID-19 pandemic on cancer-related patient encounters. JCO Clin Cancer Inform 4:657-665. https:// doi.org/10.1200/CCI.20.00068

16. Lambertini M, Toss A, Passaro A et al (2020) Cancer care during the spread of coronavirus disease 2019 (COVID-19) in Italy: young oncologists' perspective. ESMO Open 5(2):e000759. https ://doi.org/10.1136/esmoopen-2020-000759

17. Annunziata S, Bauckneht M, Albano D et al (2020) Impact of the COVID-19 pandemic in nuclear medicine departments: preliminary report of the first international survey. Eur J Nucl Med Mol Imaging. https://doi.org/10.1007/s00259-020-04874-z (published online ahead of print, 2020 May 27)

18. Tuech JJ, Gangloff A, Di Fiore F et al (2020) Strategy for the practice of digestive and oncological surgery during the Covid-19 epidemic. J Visc Surg. https://doi.org/10.1016/j.jvisc surg.2020.03.008 (published online ahead of print, 2020 Mar 31)

19. Gill S, Hao D, Hirte H, Campbell A, Colwell B (2020) Impact of COVID-19 on Canadian medical oncologists and cancer care: Canadian Association of Medical Oncologists survey report. Curr Oncol 27(2):71-74. https://doi.org/10.3747/co.27.6643

20. Chen YM, Perng RP, Chu H, Tsai CM, Whang-Peng J (2004) Impact of severe acute respiratory syndrome on the status of lung cancer chemotherapy patients and a correlation of the signs and symptoms. Lung Cancer 45(1):39-43. https://doi.org/10.1016/j. lungcan.2004.01.002

Publisher's Note Springer Nature remains neutral with regard to jurisdictional claims in published maps and institutional affiliations.

\section{Affiliations}

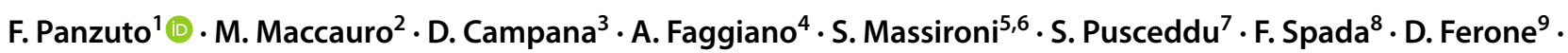
R. Modica ${ }^{10}$. C. M. Grana ${ }^{11}$ - P. Ferolla ${ }^{12} \cdot$ M. Rinzivillo ${ }^{1}$. G. Badalamenti ${ }^{13} \cdot$ M. C. Zatelli ${ }^{14} \cdot$ F. Gelsomino $^{15}$. E. De Carlo ${ }^{16} \cdot$ M. Bartolomei ${ }^{17}$ - M. P. Brizzi ${ }^{18} \cdot$ S. Cingarlini ${ }^{19} \cdot$ A. Versari ${ }^{20} \cdot$ G. Fanciulli ${ }^{21} \cdot$ E. Arvat $^{22} \cdot$ E. Merola $^{23}$. M. Cives ${ }^{24} \cdot$ S. Tafuto $^{25} \cdot$ S. Baldari ${ }^{26} \cdot$ M. Falconi ${ }^{27}$

1 Digestive Disease Unit, ENETS Center of Excellence, Sant'Andrea University Hospital, Via di Grottarossa 1035, 00189 Rome, Italy

2 Nuclear Medicine Unit, ENETS Center of Excellence, IRRCS National Cancer Institute (INT), Milan, Italy
3 Division of Oncology, Azienda Ospedaliero-Universitaria di Bologna, Bologna, Italy

4 Endocrinology Unit, Department of Experimental Medicine, Sapienza University of Rome, Rome, Italy 
5 Division of Gastroenterology and Center for Autoimmune Liver Diseases, Department of Medicine and Surgery, University of Milano-Bicocca, Monza, Italy

6 European Reference Network on Hepatological Diseases (ERN RARE-LIVER), San Gerardo Hospital, Monza, Italy

7 Oncology Unit, ENETS Center of Excellence, IRRCS National Cancer Institute (INT), Milan, Italy

8 Oncology Unit, ENETS Center of Excellence, European Cancer Institute (IEO), Milan, Italy

9 Endocrinology Unit, IRCCS Ospedale Policlinico San Martino; Endocrinology, Department of Internal Medicine and Medical Specialties (DIMI), University of Genoa, Genoa, Italy

10 Department of Clinical Medicine and Surgery, University "Federico II", Naples, Italy

11 Nuclear Medicine Division, IRCCS European Institute of Oncology, Milan, Italy

12 Multidisciplinary Group for Diagnosis and Treatment of Neuroendocrine Tumors Umbria Regional Cancer Network, Perugia, Italy

13 Department of Surgical, Oncological and Oral Sciences, Section of Medical Oncology, University of Palermo, Palermo, Italy

14 Section of Endocrinology and Internal Medicine,, Department of Medical Sciences, University of Ferrara, Ferrara, Italy

15 Department of Oncology and Hematology, University Hospital of Modena, Modena, Italy
16 Department of Medicine, Internal Medicine III, University of Padova, Padua, Italy

17 Nuclear Medicine Department, Sant'Anna Hospital, Ferrara, Italy

18 Department of Oncology, San Luigi Gonzaga Hospital, University of Turin, Orbassano, Italy

19 Oncology Unit, ENETS Center of Excellence, University Hospital of Verona, Verona, Italy

20 Nuclear Medicine Unit, Azienda Unità Sanitaria Locale-IRCCS of Reggio Emilia, Reggio Emilia, Italy

21 NET Unit, Department of Medical, Surgical and Experimental Sciences, University of Sassari - Endocrine Unit, AOU Sassari, Sassari, Italy

22 Oncological Endocrinology Unit, Department of Medical Sciences, University of Turin, Turin, Italy

23 Department of Gastroenterology, Azienda Provinciale per i Servizi Sanitari di Trento (APSS), Trento, Italy

24 Department of Biomedical Sciences and Human Oncology, University of Bari, Bari, Italy

25 S.C. Sarcomi e Tumori Rari, Istituto Nazionale Tumori, IRCCS Fondazione G. Pascale, Napoli, Italy

26 Nuclear Medicine Unit, Department of Biomedical and Dental Sciences and of Morpho-Functional Imaging, University of Messina, Messina, Italy

27 Pancreatic Surgery, ENETS Center of Excellence, Pancreas Translational and Clinical Research Center, IRCCS San Raffaele Scientific Institute, Milan, Italy 